\title{
Salmonella bongori 48:235:- The first Italian case of human infection outside Sicily
}

\author{
Alberto Bellio, ${ }^{1}$ Daniela M. Bianchi, ${ }^{1}$ Pierluigi Acutis, ${ }^{2}$ Cristina Biolatti, ${ }^{2}$ Ida Luzzi, ${ }^{3}$ \\ Paola Modesto, ${ }^{2}$ Andrea Rocchetti, ${ }^{4}$ Lucia Decastelli, ${ }^{1}$ Silvia Gallina ${ }^{5}$ \\ ${ }^{1}$ Complex Unit for Food Control and Production Hygene, and ${ }^{2}$ Genetic Laboratory and \\ Immunobiochemicals Unit, Institute for Experimental Veterinary Medicine of Piedmont, Liguria \\ and Aosta Valley, Turin; ${ }^{3}$ National Institute of Health, Rome; ${ }^{4}$ National Health Unit "SS. Antonio e \\ Biagio e C. Arrigo", Alessandria; ${ }^{5}$ Reference Centre for Salmonellae Typing, Institute for \\ Experimental Veterinary Medicine of Piedmont, Liguria and Aosta Valley, Turin, Italy
}

\section{Summary}

Salmonella bongori $48 \mathrm{z}_{35}$ :- $^{-}$is considered endemic to Sicily (Italy) due to its epidemiological peculiarity. To our knowledge, no previous cases of human infection caused by $S$. bongori $48: z_{35}$ :- have ever been reported in mainland Italy. Here we describe the isolation of $S$. bongori 48: $\mathrm{z}_{35}$ :- from a 1-year-old symptomatic child in northwest Italy (Piedmont Region). The strain showed no antimicrobial resistance. Reporting of $S$. bongori $48: \mathrm{z}_{35}$ :- in a previously safe area is important to identify epidemiological changes.

\section{Introduction}

Strains of Salmonella bongori with the antigenic formula $48: \mathrm{z}_{35}$ :have been isolated from various different sources in Sicily: 23 children

Correspondence: Alberto Bellio, SC Controllo Alimenti e Igiene delle
Produzioni, Istituto Zooprofilattico Sperimentale del Piemonte, Liguria e
Valle d'Aosta, Via Bologna 148, 10154, Turin, Italy.
E-mail: alberto.bellio@izsto.it

Key words: Salmonella bongori; Italy; 1-year-old child.

Conflict of interest: the authors declare no potential conflict of interest.

Contributions: $\mathrm{AB}$ wrote the manuscript; $\mathrm{DMB}$ assisted with strain typing; PA, PM and CB carried out molecular analyses; IL assisted with antimicrobial susceptibility; AR supervised patient admission and strain isolation; LD collected data; SG coordinated the strain characterization.

Received for publication: 14 March 2016.

Revision received: 4 May 2016.

Accepted for publication: 7 May 2016.

(C) Copyright A. Bellio et al., 2016

Licensee PAGEPress, Italy

Microbiologia Medica 2016; 31:5884

doi:10.4081/mm.2016.5884

This article is distributed under the terms of the Creative Commons Attribution Noncommercial License (by-nc 4.0) which permits any noncommercial use, distribution, and reproduction in any medium, provided the original author(s) and source are credited. (aged 1 month to 3 years), an HIV-positive patient, a healthy human carrier, foodstuffs (soft cheese and hen's eggs), pigeons, blackcaps, and urban sewerage plants. In the majority of the human cases, illness occurred in infants and toddlers, manifesting with moderate to severe diarrhea and fever. One instance of infection with $S$. bongori $48: \mathrm{z}_{35}$ :was reported in a dog with diarrhea in Calabria, Cosenza (southern Italy) in 1999. The apparently exclusive presence of $S$. bongori $48: z_{35}$ :- strains suggests that this serovar is endemically circulating in Sicily $(2,4)$.

To our knowledge, no cases of human infection with $S$. bongori $48: z_{35}$ :- have ever been reported outside Sicily. Elsewhere, the only recorded isolates were the isolation from a lizard in Chad in 1966, reported as the first isolated strain, and isolates from foodstuffs in England and Turkey $(1,4)$.

We report a recent case of infection with $S$. bongori $48: \mathrm{z}_{35}$ :- in a 1year-old child in northwest Italy.

\section{Case Report}

In August 2014, a toddler was admitted to the Hospital of Alessandria (northwest Italy) because of severe haemorrhagic diarrhoea and fever. The remainder of the physical examination was unremarkable and hydration was normal. Bleeding punctate abrasions were seen on the perianal skin. Paracetamol was given. The child was hospitalised for 2 days and discharged after remission of symptoms. Treatment with ceftibuten for 4 days and probiotics for 10 days was prescribed. Blood and stool samples were sent to the hospital's microbiology laboratory for pathogen detection. Salmonella spp. strain was isolated and identified from the stool samples.

The strain was subsequently typed as $S$. bongori $48: \mathrm{z}_{35}$ :- by the Regional Reference Laboratory for Salmonella typing according to the Kauffman-White and Le Minor scheme. S. bongori was confirmed by 16S rDNA sequencing using the MicroSEQ Full Gene system (Life Technologies). Antimicrobial resistance was verified by the disk diffusion method; the antibiotic used and their concentrations ( $\mathrm{g}$ ) were: nalidixic acid (NAL, 30), ampicillin (A, 10), cefotaxime (CTX, 5), ceftazidime (CAZ, 10), amoxicillin/clavulanic acid 2:1 (AMC, 30), meropenem (MEM, 10), chloramphenicol (C, 30), gentamicin (G, 10), kanamycin (K, 30), streptomycin ( $\mathrm{S}, 10)$, sulfonamides ( $\mathrm{Su}, 0.25)$, tetracycline (T, 30), trimethoprim (TMP, 5), and trimethoprim-sulfamethoxazole (SXT, 1.25/23.75). The reference strain Escherichia coli ATCC 25922 was used as a control for each experiment. EUCAST guidelines were applied for category interpretation of antibiotics, as 
described by Garcìa-Fernàndez (3). The strain was fully susceptible against the tested antibiotics (Table 1).

Epidemiological investigation by telephone interview with the child's mother revealed that the child and his family had recently returned by ferry from Sardinia but yielded scant clues about possible sources or routes of infection. No previous contacts with birds or cold-blooded animals were reported. No clear link between the illness and the consumption of food or drink could be established. Infection might have occurred during the family's stay in Sardinia or while aboard the ferry.

\section{Conclusions}

The prevalence of $S$. bongori is reportedly low but persistent in southern Italy (4). Unfortunately, the epidemiological investigation

Table 1. Antimicrobial susceptibility of Salmonella bongori 48:z 35 :-

\begin{tabular}{lcl} 
Antimicrobial & Concentration $(\mu \mathrm{g})$ & Susceptibility \\
Nalidixic acid & 30 & Susceptible \\
Ampicillin & 10 & Susceptible \\
\hline Cefotaxime & 5 & Susceptible \\
Caftazidime & 10 & Susceptible \\
\hline Amoxicillin/clavulanic acid 2:1 & 30 & Susceptible \\
Meropenem & 10 & Susceptible \\
\hline Chloramphenicol & 30 & Susceptible \\
Gentamicin & 10 & Susceptible \\
\hline Kanamycin & 30 & Susceptible \\
Streptomycin & 10 & Susceptible \\
\hline Sulfonamides & 0.25 & Susceptible \\
Tetracycline & 30 & Susceptible \\
\hline Trimethoprim & 5 & Susceptible \\
Trimethoprim-sulfamethoxazole & $1.25 / 23.75$ & Susceptible \\
\hline
\end{tabular}

failed to identify the source of infection in the present case, which a more detailed and accurate investigation might conceivably have resolved. Since the child's parents showed no symptoms, no biological samples were obtained for microbiological analysis. The hospital protocol does not provide for sampling asymptomatic family members when a child is admitted for gastroenteritis. However, because asymptomatic human carriers are a known source of infection, microbiological analysis of biological samples from the parents could have added useful information to solve this case (5). Furthermore, no samples of the food the child ate on the ferry were available for microbiological analysis, which, because food can be a vehicle for infection with $S$. bongori (1), could have aided in uncovering the source of infection.

Monitoring the spread of $S$. bongori $48: \mathrm{z}_{35}$ :- is needed to collect more information about this serovar, which, until now, seemed to be confined to southern Italy. Reporting the isolation of a new pathogen in a previously safe area is the first step to identify epidemiological changes and plan surveillance programs.

\section{References}

1. Arslan S, Eyi A. Occurrence and antimicrobial resistance profiles of Salmonella species in retail meat products. J Food Prot 2010;73:1613-7.

2. Foti M, Rinaldo D, Guercio A, et al. Pathogenic microorganisms carried by migratory birds passing through the territory of the island of Ustica, Sicily (Italy). Avian Path 2011;40:405-9.

3. Garcìa-Fernàndez $A$, Gallina $S$, Owczarek $S$, et al. Emergence of ciprofloxacin-resistant Salmonella enterica serovar typhi in Italy. PloS ONE 2015;10:6.

4. Giammanco GM, Pignato S, Mammina C, et al. Persistent endemicity of Salmonella bongori $48: z_{35}$ :- in southern Italy: molecular characterization of human, animal and environmental isolates. J Clin Microbiol 2002;40:3502-5.

5. Marineli F, Tsoucalas G, Karamanou M, Androutsos G. Mary Mallon (1869-1938) and the history of typhoid fever. Ann Gastroenterol 2013;26:132-4. 